\title{
Spacelike developable surfaces through a common line of curvature in Minkowski 3-space
}

\author{
Cai-Yun $\mathrm{LI}^{*}$, Chun-Gang ZHU** and Ren-Hong WANG** \\ * School of Science, Dalian University of Technology \\ No.2 Dagong Road, Liaodong Bay District, Panjin, 124221, China. \\ ** School of Mathematical Sciences, Dalian University of Technology \\ No.2 Linggong Road, Ganjingzi District, Dalian, 116024, China. \\ E-mail: cgzhu@dlut.edu.cn
}

Received 23 December 2014

\begin{abstract}
This paper studies the problem to construct a spacelike developable surface possessing a given non-null parametric curve as the line of curvature in Minkowski 3-space. By using Frenet frame to express the surface, we derive the necessary and sufficient conditions when the resulting spacelike developable surface is cylinder, cone or tangent surface. Finally, we give some representative examples.
\end{abstract}

Key words : Spacelike developable surface, Line of curvature, Frenet frame

\section{Introduction}

In $E^{3}$ (Euclidean 3-space), developable surfaces are a subset of ruled surfaces. They can be developed onto a plane without streching and tearing. Therefore, they have important application in many product design which use leather, paper, sheet metal as materials. There are three types of developable surfaces: cylinders, cones and tangent surfaces.

There are mainly two methods for the design of developable surfaces. One approach is to prove characterizing equations for the free form surfaces to be developable. The readers can refer to papers (Nolan, 1971, Aumann, 1991, Chalfant and Maekawa, 1998). However, the complex system of coupled equations is very difficult for the design of developable Bézier surfaces in a CAD system. Another approach to design developable surfaces is a direct surface represented in terms of geometric duality between points and planes in 3D projective space by Chu and Séquin (Chu and Séquin, 2002). In paper (Bodduluri and Ravani, 1993), the concept of duality between points and planes in 3D projective space was used to develop a new representation for developable surfaces in terms of plane geometry.

Line of curvature is one of the most important characteristic curve on a surface and it plays an important role in differential geometry, please refer to the book by Carmo (1976) and Willmore (1959). It is a useful tool in surface analysis for exhibiting variations of the principal direction. There are a number of papers studied the lines of curvature. Martin (Martin, 1983) introduced the principal patches whose sides are lines of curvature. He showed that the existence of such patches was contingent upon certain position matching and frame matching conditions along the patch boundary curves. Alourdas et al. (Alourdas, et al., 1990) studied how to construct a net of lines of curvature on a B-spline surface. In paper (Li, et al., 2011), the authors studied the parametric surface pencils which possess the given curve as the line of curvature. Moreover, the authors further gave an approach to design the developable surface through the given curve as line of curvature in paper ( $\mathrm{Li}$, et al., 2013).

As we all know, Minkowski geometry provides the theoretical model in mathematics for Einstein's relativity theory. Especially, $E_{1}^{4}$ (Minkowski 4-space) has the very strong physical background. As the sub-space of $E_{1}^{4}, E_{1}^{3}$ (Minkowski 3 -space) has many properties which are different from $E^{3}$. Some basic concepts, such as vector, frame, and the motion of point, have qualitative changes. Since the metric in $E_{1}^{3}$ is Lorentzian metric which is not positive definite metric. The distance function in $E_{1}^{3}$ can be positive, negative or zero. Therefore, the vectors in $E_{1}^{3}$ can be classified into three types: spacelike vectors, timelike vectors and lightlike vectors according to the sign of the distance function. Especially, 
the emergence of lightlike vectors makes the results of some problems are surprising. As the spatio-temporal model of relativity theory, Minkowski space obtains much attention in the field of mathematics and physics. The theory of submanifold in Minkowski space has been an important topic in the field of geometry. In this paper, we continue the research of the paper (Li, et al., 2013). We give an approach to design the spacelike developable surface which possess the given curve as line of curvature, and we also derive the necessary and sufficient conditions for the spacelike developable surface to be cylinder, cone or tangent surface respectively.

The paper is organized as follows. In Section 2, we briefly recall the relevant definitions and results about curve and surface of Differential Geometry in $E_{1}^{3}$. In Section 3, for a given non-null parametric curve $\boldsymbol{r}(s)$, where $s$ is arc-length parameter, we construct a spacelike developable surface which possesses $\boldsymbol{r}(s)$ as the line of curvature and give the concrete expression of the resulting surface. Moreover, we derive the conditions when the developable surface is cylinder, cone or tangent surface respectively. Some representative examples are given in Section 4. Finally, we conclude the paper in Section 5 .

\section{Backgrounds}

\subsection{Curves and surfaces in Minkowski 3-space}

Definition 1 (Lopez, 2008)The Lorentz-Minkowski space is the metric space $E_{1}^{3}=\left(R^{3},<,>\right)$, where the metric $<$,> is given by

$$
<\boldsymbol{u}, \boldsymbol{v}>=u_{1} v_{1}+u_{2} v_{2}-u_{3} v_{3}, \quad \boldsymbol{u}=\left(u_{1}, u_{2}, u_{3}\right), \boldsymbol{v}=\left(v_{1}, v_{2}, v_{3}\right)
$$

The metric $<,>$ is called the Lorentzian metric.

Definition 2 (Lopez, 2008)A vector $v \in E_{1}^{3}$ is called

(1) spacelike if $\langle\boldsymbol{v}, \boldsymbol{v}\rangle>0$ or $\boldsymbol{v}=0$,

(2) timelike if $\langle\boldsymbol{v}, \boldsymbol{v}\rangle<0$ and

(3) lightlike if $\langle\boldsymbol{v}, \boldsymbol{v}\rangle=0$ and $\boldsymbol{v} \neq 0$.

We point out that the null vector $\boldsymbol{v}=0$ is considered of spacelike type although it satisfies $<\boldsymbol{v}, \boldsymbol{v}\rangle=0$.

The definition of vector product is the same as the given one in the Euclidean ambient.

Definition 3 (Lopez, 2008)If $\boldsymbol{u}, \boldsymbol{v} \in E_{1}^{3}$, we call the Lorentzian vector product of $\boldsymbol{u}$ and $\boldsymbol{v}$ to the unique vector denoted by $\boldsymbol{u} \times \boldsymbol{v}$ that satisfies

$<\boldsymbol{u} \times \boldsymbol{v}, \boldsymbol{w}>=\operatorname{det}(\boldsymbol{u}, \boldsymbol{v}, \boldsymbol{w})$,

where $\operatorname{det}(\boldsymbol{u}, \boldsymbol{v}, \boldsymbol{w})$ is the determinant of the matrix obtained by putting by columns the coordinates of the three vectors $\boldsymbol{u}, \boldsymbol{v}$ and $\boldsymbol{w}$.

For any two vectors $\boldsymbol{u}=\left(u_{1}, u_{2}, u_{3}\right)$ and $\boldsymbol{v}=\left(v_{1}, v_{2}, v_{3}\right)$, the vector product is defined by $\boldsymbol{u} \times \boldsymbol{v}=\left(u_{2} v_{3}-u_{3} v_{2}, u_{3} v_{1}-\right.$ $\left.u_{1} v_{3},-\left(u_{1} v_{2}-u_{2} v_{1}\right)\right)$.

Let $\boldsymbol{r}(s)$ be a curve in $E_{1}^{3}$, where $s$ is the arc-length parameter. We say that $\boldsymbol{r}(s)$ is spacelike (resp. timelike, lightlike) at $s$ if $\dot{\boldsymbol{r}}(s)$ is a spacelike (resp. timelike, lightlike) vector. In this paper, $\dot{\boldsymbol{r}}(s)$ denote the derivatives of $\boldsymbol{r}$ with respect to arc length parameter $s$. The curve $\boldsymbol{r}(s)$ is called spacelike (resp. timelike, lightlike) if it is for any $s \in I$.

We suppose that $\boldsymbol{r}(s)$ be a unit speed timelike curve in $E_{1}^{3}$. Let $k(s)$ and $\tau(s)$ be the curvature and torsion. Assume $\ddot{\boldsymbol{r}}(s) \neq 0$ for all $s \in[0, L]$, since this would give us a straight line. For each point of $\boldsymbol{r}(s)$, the corresponding Serret-Frenet formulae is given by (Walfare 1995):

$$
\begin{aligned}
\dot{\boldsymbol{T}}(s) & =k(s) \boldsymbol{N}(s) \\
\dot{\boldsymbol{N}}(s) & =k(s) \boldsymbol{T}(s)+\tau(s) \boldsymbol{B}(s), \\
\dot{\boldsymbol{B}}(s) & =-\tau(s) \boldsymbol{N}(s)
\end{aligned}
$$

and

$$
\boldsymbol{T}(s) \times \boldsymbol{N}(s)=\boldsymbol{B}(s), \boldsymbol{T}(s) \times \boldsymbol{B}(s)=-\boldsymbol{N}(s), \boldsymbol{N}(s) \times \boldsymbol{B}(s)=-\boldsymbol{T}(s) .
$$

If $\boldsymbol{r}(s)$ is a unit speed spacelike curve, the Serret-Frenet formulaes are as follows: 
( 1 ) $\boldsymbol{N}(s)$ is spacelike and $\boldsymbol{B}(s)$ is timelike

$$
\begin{aligned}
& \dot{\boldsymbol{T}}(s)=k(s) \boldsymbol{N}(s), \\
& \dot{\boldsymbol{N}}(s)=-k(s) \boldsymbol{T}(s)+\tau(s) \boldsymbol{B}(s), \\
& \dot{\boldsymbol{B}}(s)=\tau(s) \boldsymbol{N}(s),
\end{aligned}
$$

and

$$
\boldsymbol{T}(s) \times N(s)=-\boldsymbol{B}(s), \boldsymbol{T}(s) \times \boldsymbol{B}(s)=-\boldsymbol{N}(s), \boldsymbol{N}(s) \times \boldsymbol{B}(s)=\boldsymbol{T}(s) .
$$

(2) $\boldsymbol{N}(s)$ is timelike and $\boldsymbol{B}(s)$ is spacelike

$$
\begin{aligned}
& \dot{\boldsymbol{T}}(s)=k(s) \boldsymbol{N}(s), \\
& \dot{\boldsymbol{N}}(s)=k(s) \boldsymbol{T}(s)+\tau(s) \boldsymbol{B}(s), \\
& \dot{\boldsymbol{B}}(s)=\tau(s) \boldsymbol{N}(s),
\end{aligned}
$$

and

$$
\boldsymbol{T}(s) \times \boldsymbol{N}(s)=\boldsymbol{B}(s), \boldsymbol{T}(s) \times \boldsymbol{B}(s)=\boldsymbol{N}(s), \boldsymbol{N}(s) \times \boldsymbol{B}(s)=\boldsymbol{T}(s) .
$$

Here $\boldsymbol{N}(s)$ and $\boldsymbol{B}(s)$ are called the normal vector and the binormal vector respectively.

For a parametric surface $\boldsymbol{P}(s, t)$, it is called a spacelike (timelike) if the induced metric on $\boldsymbol{P}(s, t)$ is a Riemannian (Lorentzian) metric on each tangent plane. That is to say, the unit normal vector $\boldsymbol{n}(s, t)$ of the surface $\boldsymbol{P}(s, t)$ is timelike (spacelike) on each point of $\boldsymbol{P}(s, t)$, where

$$
\boldsymbol{n}(s, t)=\frac{\boldsymbol{P}_{s}(s, t) \times \boldsymbol{P}_{t}(s, t)}{\left\|\boldsymbol{P}_{s}(s, t) \times \boldsymbol{P}_{t}(s, t)\right\|} .
$$

\subsection{Ruled surface}

Ruled surface is the well-known geometric object. Extending the research of ruled surface from $E^{3}$ to $E_{1}^{3}$, and studying its geometric properties is an important issue. De Woestijne (de Woestijne, 1990) classified all ruled surfaces in $E_{1}^{3}$. Kim and Yoon (Kim and Yoon, 2004) classified the ruled surfaces satisfying Gauss map conditions in $E_{1}^{3}$.

Now, we define a ruled surface $M$ in $E_{1}^{3}$. Let $\alpha=\alpha(s)$ be a curve defined on an open interval $I_{1}$ of $R$ and $\beta=\beta(s)$ be a transversal vector field along $\alpha$. For an open interval $I_{2}$ of $R$, we have the parametrization for $M$

$$
\boldsymbol{P}(s, t)=\alpha(s)+t \beta(s), \quad s \in I_{1}, t \in I_{2},
$$

where $\alpha(s)$ is called a base curve and $\beta(s)$ a director curve.

For non-developable ruled surface (15) in $E_{1}^{3}$, if $\left\langle\alpha^{\prime}, \alpha^{\prime}\right\rangle \neq 0$ and $\left\langle\alpha^{\prime}, \beta\right\rangle=0$, then the ruled surface (15) is said to be of type $M_{+}$or type $M_{-}$.

(1) If $\left\langle\alpha^{\prime}, \alpha^{\prime}>>0\right.$ and $\left\langle\beta, \beta>=1\right.$, when $\left\langle\beta^{\prime}, \beta^{\prime}><0\left(<\beta^{\prime}, \beta^{\prime}>=0\right)\right.$, the ruled surface (15) is said to be type $M_{+}^{1}\left(M_{+}^{2}\right)$. If $\left\langle\alpha^{\prime}, \alpha^{\prime}>>0\right.$ and $\left\langle\beta, \beta>=-1\right.$, when $\left\langle\beta^{\prime}, \beta^{\prime}>>0\right.$, the ruled surface (15) is said to be type $M_{+}^{3}$.

(2) If $\left\langle\alpha^{\prime}, \alpha^{\prime}><0\right.$ and $<\beta, \beta>=1$, when $\left\langle\beta^{\prime}, \beta^{\prime}><0\left(<\beta^{\prime}, \beta^{\prime}>=0\right)\right.$, the ruled surface (15) is said to be type $M_{-}^{1}\left(M_{-}^{2}\right)$.

In the following, we give a simple example.

Example 1 Let a be a nonzero constant, the ruled surface $M$ in $E_{1}^{3}$ can be expressed

$$
\boldsymbol{P}(s, t)=(a s, t \cos s, t \sin s) .
$$

We choose $a=2$, this parametrization defines a non-developable ruled surface of type $M_{-}^{1}$ in $E_{1}^{3}$, which is called a helicoid of the 3rd kind. See Fig.1.

\section{Construction of spacelike developable surface with a non-null line of curvature}

Firstly, we review a known theorem, which characterizes lines of curvature on a surface.

Theorem 1 A curve on a surface is a line of curvature if and only if the surface normals along the curve form a developable surface. 


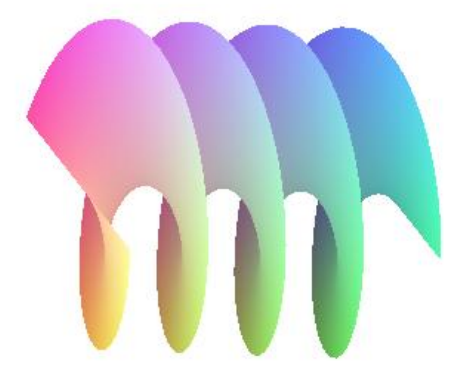

Fig. 1 Helicoid of the 3rd kind.

\subsection{The given curve $r(s)$ is spacelike curve}

(1) $\boldsymbol{N}(s)$ is spacelike, and $\boldsymbol{B}(s)$ is timelike.

Let the normal surface of $\boldsymbol{r}(s)$ be

$$
\boldsymbol{Q}(s, t)=\boldsymbol{r}(s)+\boldsymbol{t n}_{1}(s),
$$

where $\boldsymbol{n}_{1}(s)=\boldsymbol{N} \sinh \theta+\boldsymbol{B} \cosh \theta$ is timelike. Let $\boldsymbol{b}_{1}(s)=\boldsymbol{N} \cosh \theta+\boldsymbol{B} \sinh \theta$.

According to the Theorem 1, $\boldsymbol{r}(s)$ is the line of curvature of the surface $\boldsymbol{P}(s, t)$ if and only if $\boldsymbol{Q}(s, t)$ is developable and $\boldsymbol{n}_{1}(s)$ is the normal vector of $\boldsymbol{P}(s, t)$. By computing, we have

$$
\begin{aligned}
\dot{\boldsymbol{n}}_{1}(s) & =\dot{\theta} \cosh \theta \boldsymbol{N}+\sinh \theta \dot{\boldsymbol{N}}+\dot{\theta} \sinh \theta \boldsymbol{B}+\cosh \theta \dot{\boldsymbol{B}} \\
& =\dot{\theta} \cosh \theta \boldsymbol{N}+\sinh \theta(-k \boldsymbol{T}+\tau \boldsymbol{B})+\dot{\theta} \sinh \theta \boldsymbol{B}+\tau \cosh \theta \boldsymbol{N} \\
& =-k \sinh \theta \boldsymbol{T}+(\dot{\theta}+\tau) \boldsymbol{b}_{1} \\
\dot{\boldsymbol{b}}_{1}(s) & =\dot{\theta} \sinh \theta \boldsymbol{N}+\cosh \theta \dot{\boldsymbol{N}}+\dot{\theta} \cosh \theta \boldsymbol{B}+\sinh \theta \dot{\boldsymbol{b}}_{1} \\
& =\dot{\theta} \sinh \theta \boldsymbol{N}+\cosh \theta(-k \boldsymbol{T}+\tau \boldsymbol{B})+\dot{\theta} \cosh \theta \boldsymbol{B}+\tau \sinh \theta \boldsymbol{N} \\
& =-k \cosh \theta \boldsymbol{T}+(\dot{\theta}+\tau) \boldsymbol{n}_{1}
\end{aligned}
$$

Then

$$
\begin{aligned}
\left(\dot{\boldsymbol{r}}, \boldsymbol{n}_{1}, \dot{\boldsymbol{n}}_{1}\right)=0 & \Longleftrightarrow\left(\boldsymbol{T}, \sinh \theta \boldsymbol{N}+\cosh \theta \boldsymbol{B},-k \sinh \theta \boldsymbol{T}+(\dot{\theta}+\tau) \boldsymbol{b}_{1}\right)=0 \\
& \Longleftrightarrow\left(\boldsymbol{T}, \sinh \theta \boldsymbol{N},(\dot{\theta}+\tau) \boldsymbol{b}_{1}\right)+\left(\boldsymbol{T}, \cosh \theta \boldsymbol{B},(\dot{\theta}+\tau) \boldsymbol{b}_{1}\right)=0 \\
& \Longleftrightarrow \dot{\theta}+\tau=0 \\
& \Longleftrightarrow \dot{\theta}=-\tau .
\end{aligned}
$$

That is

$$
\theta=-\int_{s_{0}}^{s} \tau d s+\theta_{0}
$$

where $s_{0}$ is the starting value of the arc length. Generally, we choose $s_{0}=0$, and then $\theta_{0}=\theta(0)$.

Following, we give the corresponding theorem of the spacelike developable surface possessing the given spacelike curve as a line of curvature.

Theorem 2 Given a spacial parametric spacelike curve $\boldsymbol{r}(s)$, there exists a spacelike developable surface $\boldsymbol{P}(s, t)$ possessing $\boldsymbol{r}(s)$ as a line of curvature.

Proof For the spacelike curve $\boldsymbol{r}(s)$, suppose the normal surface of it be

$$
\boldsymbol{P}_{1}(s, t)=\boldsymbol{r}(s)+\operatorname{tn}_{1}(s)
$$

where $\boldsymbol{n}_{1}(s)=\boldsymbol{N}(s) \sinh \theta+\boldsymbol{B}(s) \cosh \theta$ is timelike. $\theta(s)$ is defined by Eq.(17). Define a surface

$$
\boldsymbol{P}(s, t)=\boldsymbol{r}(s)-t \boldsymbol{b}_{1}(s),
$$

obviously, $\boldsymbol{P}(s, 0)=\boldsymbol{r}(s)$. Since

$$
\begin{aligned}
\boldsymbol{P}_{s}(s, t) \times\left.\boldsymbol{P}_{t}(s, t)\right|_{t=0} & =\left(\dot{\boldsymbol{r}}(s)-t \dot{\boldsymbol{b}}_{1}(s)\right) \times\left.\left(-\boldsymbol{b}_{1}(s)\right)\right|_{t=0} \\
& =-\boldsymbol{T}(s) \times(\cosh \theta \boldsymbol{N}+\sinh \theta \boldsymbol{B}) \\
& =\cosh \theta \boldsymbol{B}+\sinh \theta \boldsymbol{N} \\
& =\boldsymbol{n}_{1}(s),
\end{aligned}
$$


then $\boldsymbol{P}_{1}(s, t)$ is the normal surface of $\boldsymbol{P}(s, t)$. Moreover, $\theta(s)$ is defined as Eq.(17), then the surface $\boldsymbol{P}_{1}(s, t)$ is developable. According to Theorem $1, \boldsymbol{r}(s)$ is a line of curvature of $\boldsymbol{P}(s, t)$.

Finally, since

$$
\left(\dot{\boldsymbol{r}},-\boldsymbol{b}_{1},-\dot{\boldsymbol{b}}_{1}\right)=\left(\boldsymbol{T}, \boldsymbol{b}_{1}, k \cosh \theta \boldsymbol{T}\right)=0,
$$

the spacelike surface $\boldsymbol{P}(s, t)$ is developable. The conclusion holds.

In the following, we derive the conditions when the spacelike developable surface (19) is cylinder, cone or tangent surface respectively.

Theorem 3 The spacelike developable surface Eq.(19) is a cylinder if and only if $\theta=0$.

Proof The developable surface Eq.(19) is cylinder if and only if $\boldsymbol{b}_{1} \times \dot{\boldsymbol{b}}_{1}=0$. By computing,

$$
\begin{aligned}
\boldsymbol{b}_{1} \times \dot{\boldsymbol{b}}_{1} & =(\cosh \theta \boldsymbol{N}+\sinh \theta \boldsymbol{B}) \times(-k \sinh \theta \boldsymbol{T}) \\
& =-k \sinh \theta \cosh \theta(\boldsymbol{N} \times \boldsymbol{T})-k \sinh ^{2} \theta(\boldsymbol{B} \times \boldsymbol{T}) \\
& =-k \sinh \theta \cosh \theta \boldsymbol{B}-k \sinh ^{2} \theta \boldsymbol{N} .
\end{aligned}
$$

That is

$$
\begin{aligned}
\boldsymbol{b}_{1} \times \dot{\boldsymbol{b}}_{1}=0 & \Longleftrightarrow\left\{\begin{array}{l}
k \sinh \theta \cosh \theta=0 \\
k \sinh ^{2} \theta=0
\end{array}\right. \\
& \Longleftrightarrow \sinh \theta=0 \\
& \Longleftrightarrow \theta=0 .
\end{aligned}
$$

This ends the proof.

Theorem 4 The spacelike developable surface Eq.(19) is a cone if and only if $k \cosh \theta=k_{0} \cosh \theta_{0}$, where $\theta_{0}=\theta(0)$, $k_{0}=$ $k(0)$.

Proof Let $\boldsymbol{w}(s)=\boldsymbol{r}(s)-\frac{\dot{\boldsymbol{r}}(s) \cdot \dot{\boldsymbol{b}}_{1}(s)}{\dot{\boldsymbol{b}}_{1}(s) \cdot \dot{\boldsymbol{b}}_{1}(s)} \boldsymbol{b}_{1}(s)$ be the line of striction of the surface Eq.(19). Then $\boldsymbol{P}(s, t)$ is a cone if and only if

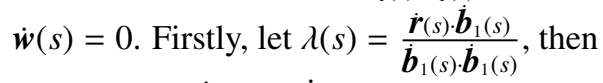

$$
\begin{aligned}
\dot{\boldsymbol{w}} & =\dot{\boldsymbol{r}}-\dot{\lambda} \boldsymbol{b}_{1}-\lambda \dot{\boldsymbol{b}}_{1} \\
& =\boldsymbol{T}-\dot{\lambda}(\cosh \theta \boldsymbol{N}+\sinh \theta \boldsymbol{B})+k \lambda \cosh \theta \boldsymbol{T} \\
& =(1+k \lambda \cosh \theta) \boldsymbol{T}-\dot{\lambda} \cosh \theta \boldsymbol{N}-\dot{\lambda} \sinh \theta \boldsymbol{B},
\end{aligned}
$$

this means $\dot{\boldsymbol{w}}=0$ if and only if

$$
\left\{\begin{array}{l}
1+\lambda k \cosh \theta=0, \\
\dot{\lambda} \cosh \theta=0, \\
\dot{\lambda} \sinh \theta=0,
\end{array}\right.
$$

i.e.

$$
\lambda=\text { const. }=-\frac{1}{k \cosh \theta} .
$$

This equivalent to

$$
k \cosh \theta=k_{0} \cosh \theta_{0},
$$

which ends the proof.

Theorem 5 The developable surface Eq.(19) is a tangent surface if and only if $k \cosh \theta \neq k_{0} \cosh \theta_{0}$, where $\theta_{0}=$ $\theta(0), k_{0}=k(0)$.

Proof According to the proof of Theorem 4, if $k \sin \theta \neq k_{0} \sin \theta_{0}$, then $\dot{\boldsymbol{w}}(s) \neq 0$. Since $\left(\dot{\boldsymbol{w}}, \boldsymbol{b}_{1}, \dot{\boldsymbol{b}}_{1}\right)=0, \dot{\boldsymbol{w}} \cdot \dot{\boldsymbol{b}}_{1}=0$ and $\left|\boldsymbol{b}_{1}\right|=1, \boldsymbol{b}_{1} \perp \dot{\boldsymbol{b}}_{1}$, we can get $\dot{\boldsymbol{w}} \| \boldsymbol{b}_{1}$. That is to say the surface Eq.(19) is a tangent surface. The conclusion holds.

(2) $\boldsymbol{N}(s)$ is timelike, and $\boldsymbol{B}(s)$ is spacelike

It is similar to the above case, we will not to analyze this situation in detail. In this case, the spacelike developable surface can be expressed as

$$
\boldsymbol{P}(s, t)=\boldsymbol{r}(s)+t \boldsymbol{b}_{1}(s)
$$

where $\boldsymbol{b}_{1}(s)=\sinh \theta \boldsymbol{N}(s)+\cosh \theta \boldsymbol{B}(s)$ and its unit normal vector is $\boldsymbol{n}_{1}(s)=\cosh \theta \boldsymbol{N}(s)+\sinh \theta \boldsymbol{B}(s)$.

The corresponding conditions when the spacelike developable surface is cylinder, cone and tangent surface respectively are as follows.

Theorem 6The spacelike developable surface Eq.(21) is a cylinder if and only if $\theta=0$.

Theorem 7The spacelike developable surface Eq.(21) is a cone if and only if $k \sinh \theta=k_{0} \sinh \theta_{0}$, where $\theta_{0}=\theta(0), k_{0}=$ $k(0)$. 
Theorem 8The developable surface Eq.(21) is a tangent surface if and only if $k \sinh \theta \neq k_{0} \sinh \theta_{0}$, where $\theta_{0}=\theta(0)$, $k_{0}=$ $k(0)$.

\subsection{The given curve $r(s)$ is timelike curve}

If the given curve $\boldsymbol{r}(s)$ is timelike curve, in order to construct a spacelike developable surface, the normal vector of the surface must be timelike. Suppose the normal vector is

$\boldsymbol{n}_{1}(s)=A_{1}(s) \boldsymbol{N}(s)+A_{2}(s) \boldsymbol{B}(s)$

where $N(s), \boldsymbol{B}(s)$ are all spacelike. Then $\left\langle\boldsymbol{n}_{1}(s), \boldsymbol{n}_{1}(s)>=A_{1}(s)^{2}+A_{2}(s)^{2}>0\right.$, which is impossible timelike. In a word, if we want to construct a spacelike surface, the curve $\boldsymbol{r}(s)$ can not be timelike.

In above theorems, we not only prove the existence of the spacelike developable surface, but also give the concrete expression of the surface. This is very meaningful in practical application.

\section{Examples}

In this section, some representative examples are given in order to verify the validity of this constructing method. In the first two examples, the curves possess spacelike normal vectors and timelike binormal vectors.

Example 2In this example, we will construct a spacelike developable surface possessing the given parametric spacelike helix as a line of curvature. Given a parametric spacelike helix,

$$
\boldsymbol{r}(s)=\left(a \cosh \left(\frac{s}{c}\right), \frac{b s}{c}, a \sinh \left(\frac{s}{c}\right)\right), a>0, b \neq 0, b^{2}-a^{2}=c^{2},-1 \leq s \leq 1 .
$$

By computing, we get

$$
\begin{aligned}
\boldsymbol{T}(s) & =\left(\frac{a}{c} \sinh \left(\frac{s}{c}\right), \frac{b}{c}, \frac{a}{c} \cosh \left(\frac{s}{c}\right)\right) \\
\boldsymbol{N}(s) & =\left(\cosh \left(\frac{s}{c}\right), 0, \sinh \left(\frac{s}{c}\right)\right), \\
\boldsymbol{B}(s) & =\left(-\frac{b}{c} \sinh \left(\frac{s}{c}\right),-\frac{a}{c},-\frac{b}{c} \cosh \left(\frac{s}{c}\right)\right), \\
k(s) & =\frac{a}{c^{2}}, \tau(s)=-\frac{b}{c^{2}} .
\end{aligned}
$$

Then $\theta(s)=\frac{b}{c^{2}} s+\theta_{0}$. If we choose $\theta_{0}=0$, the corresponding spacelike developable surface is

$$
\begin{aligned}
\boldsymbol{P}(s, t)= & \boldsymbol{r}(s)-t(\cosh \theta \boldsymbol{N}(s)+\sinh \theta \boldsymbol{B}(s)) \\
= & \left(a \cosh \left(\frac{s}{c}\right)-t \cosh \left(\frac{b s}{c^{2}}\right) \cosh \left(\frac{s}{c}\right)+\frac{b}{c} t \sinh \left(\frac{b s}{c^{2}}\right) \sinh \left(\frac{s}{c}\right), \frac{b s}{c}+\frac{a}{c} t \sinh \left(\frac{b s}{c^{2}}\right),\right. \\
& \left.a \sinh \frac{s}{c}-t \cosh \left(\frac{b s}{c^{2}}\right) \sinh \frac{s}{c}+\frac{b}{c} t \sinh \left(\frac{b s}{c^{2}}\right) \cosh \frac{s}{c}\right) .
\end{aligned}
$$

According to Theorem 5, it is a spacelike tangent developable surface. Choosing $a=1, b=2$, the surface is shown in Fig. 2(a). Figure 2(b). shows the surface with $\theta_{0}=\frac{1}{2}$.

Example 3Given a parametric spacelike circle, whose normal vector and binormal vector are spacelike and timelike respectively.

$$
\boldsymbol{r}(s)=(\cos s, \sin s, 0), \quad 0 \leq s \leq 2 \pi .
$$

After simple computation, we have

$$
\boldsymbol{T}(s)=(-\sin s, \cos s, 0), N(s)=(-\cos s,-\sin s, 0), \boldsymbol{B}(s)=(0,0,-1),
$$

and $k(s)=1, \tau(s)=0$, then $\theta(s)$ is a constant. Choosing $\theta(s)=\frac{\pi}{3}$, obviously, according to Theorem 4 , the spacelike developable surface

$$
\boldsymbol{P}(s, t)=(\cos s+t \cosh \theta \cos s, \sin s+t \cosh \theta \sin s, \sinh \theta),
$$

is a cone which possesses the given circle as line of curvature. (See Fig. 3). 


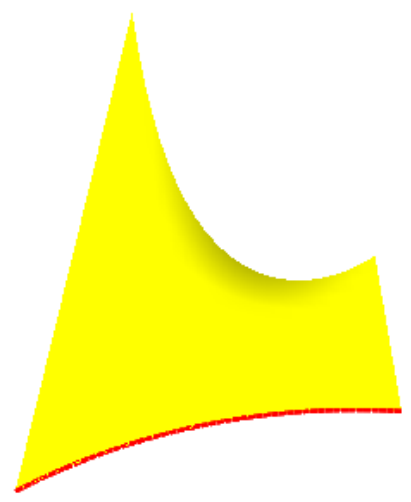

(a) The surface $\boldsymbol{P}(s, t)$ for $\theta_{0}=0$

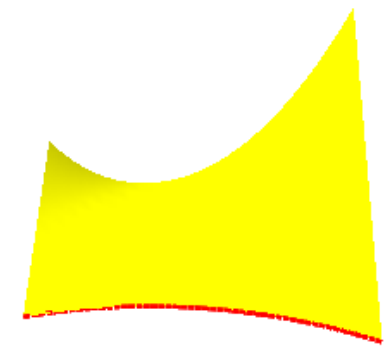

(b) The surface $\boldsymbol{P}(s, t)$ for $\theta=\frac{1}{2}$

Fig. 2 The spacelike developable surface with spacelike helix.

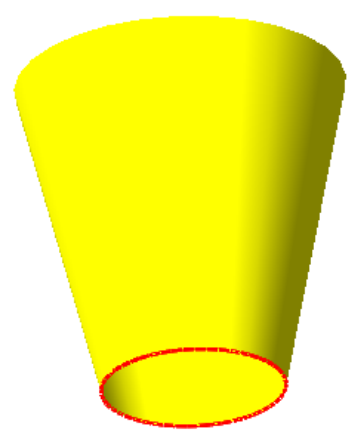

Fig. 3 The spacelike cone with the spacelike circle.

In the following examples, the normal vectors and binormal vectors of the given curves are timelike and spacelike respectively.

Example 4Given a parametric spacelike helix with timelike normal vector and spacelike binormal vector, the helix is

$$
\boldsymbol{r}(s)=\left(a \sinh \left(\frac{s}{c}\right), \frac{b s}{c}, a \cosh \left(\frac{s}{c}\right)\right), \quad-1 \leq s \leq 1,
$$

where $a, b, c \in R, a^{2}+b^{2}=c^{2}$. It is easy to get

$$
\begin{aligned}
\boldsymbol{T}(s) & =\left(\frac{a}{c} \cosh \left(\frac{s}{c}\right), \frac{b}{c}, \frac{a}{c} \sinh \left(\frac{s}{c}\right)\right) \\
N(s) & =\left(\sinh \left(\frac{s}{c}\right), 0, \cosh \left(\frac{s}{c}\right)\right), \\
\boldsymbol{B}(s) & =\left(\frac{b}{c} \cosh \left(\frac{s}{c}\right),-\frac{a}{c}, \frac{b}{c} \sinh \left(\frac{s}{c}\right)\right), \\
k(s) & =\frac{a}{c^{2}}, \tau(s)=\frac{b}{c^{2}} .
\end{aligned}
$$

Then $\theta(s)=-\frac{b}{c^{2}} s+\theta_{0}$. Let $\theta_{0}=0$, the corresponding spacelike developable surface can be expressed as

$$
\begin{aligned}
\boldsymbol{P}(s, t) & =\boldsymbol{r}(s)+t(\sinh \theta \boldsymbol{N}(s)+\cosh \theta \boldsymbol{B}(s)) \\
& =\left(a \sinh \left(\frac{s}{c}\right)+t \sinh \left(-\frac{b s}{c^{2}}\right) \sinh \left(\frac{s}{c}\right)+\frac{b}{c} t \cosh \left(-\frac{b s}{c^{2}}\right) \cosh \left(\frac{s}{c}\right), \frac{b s}{c}-\frac{a}{c} t \cosh \left(-\frac{b s}{c^{2}}\right),\right.
\end{aligned}
$$




$$
\left.a \cosh \left(\frac{s}{c}\right)+t \sinh \left(-\frac{b s}{c^{2}}\right) \cosh \left(\frac{s}{c}\right)+\frac{b}{c} t \cosh \left(\frac{b s}{c^{2}}\right) \sinh \left(\frac{s}{c}\right)\right)
$$

It is a spacelike tangent developable surface. Choosing $a=2, b=1$, the surface is shown in Fig. $4(a)$. When $\theta_{0}=\frac{1}{2}$, see Fig. 4(b).

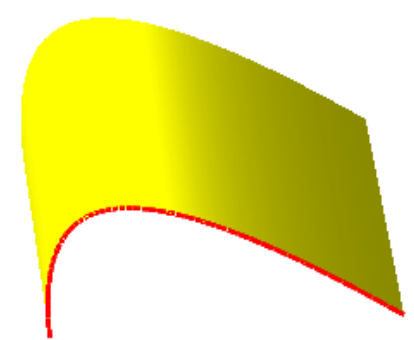

(a) The surface $\boldsymbol{P}(s, t)$ for $\theta_{0}=0$

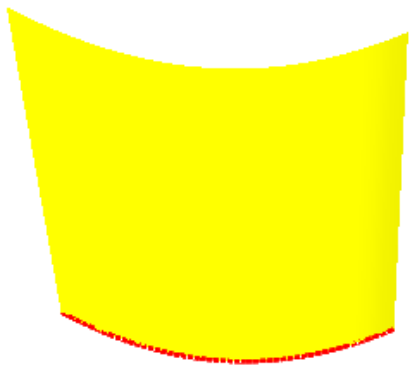

(b) The surface $\boldsymbol{P}(s, t)$ for $\theta_{0}=\frac{1}{2}$

Fig. 4 The spacelike developable surface with spacelike helix.

Example 5Suppose we are given a parametric spacelike curve

$$
\boldsymbol{r}(s)=(\sinh s, 0, \cosh s), \quad-1 \leq s \leq 1 .
$$

It is easy to get

$$
\boldsymbol{T}(s)=(\cosh s, 0, \sinh s), \boldsymbol{N}(s)=(\sinh s, 0, \cosh s), \boldsymbol{B}(s)=(0,-1,0),
$$

and $k(s)=1, \tau(s)=0$, then $\theta(s)$ is a constant. If $\theta(s)=0$, the corresponding spacelike developable surface

$$
\begin{aligned}
\boldsymbol{P}(s, t) & =\boldsymbol{r}(s)+t(\sinh \theta \boldsymbol{N}(s)+\cosh \theta \boldsymbol{B}(s)) \\
& =(\sinh s,-t, \cosh s)
\end{aligned}
$$

is a cylinder, which is shown in Fig. 5(a). Figure 5(b). shows the surface with $\theta(s)=1$.

\section{Conclusions}

In this paper, we extend our work in paper (Li 2013) to $E_{1}^{3}$ (Minkowski 3-space), and give an approach to construct a spacelike developable surface which possesses the given curve as a line of curvature. We not only prove the existence of the spacelike developable surface but also give the concrete expression of the resulting surface. At the same time, we derive the necessary and sufficient conditions when the developable surface is cylinder, cone or tangent surface respectively.

\section{Acknowledgements}

The authors appreciate the comments and valuable suggestions from the anonymous reviewers. This work is partly supported by the National Natural Science Foundation of China (Nos. 11401077, 11271060, 11290143), Fundamental Research of Civil Aircraft (No. MJ-F-2012-04), the Program for Liaoning Excellent Talents in University(No.LJQ2014010), and the Fundamental Research Funds for the Central Universities(Nos. DUT15LK47, DUT14YQ111). 


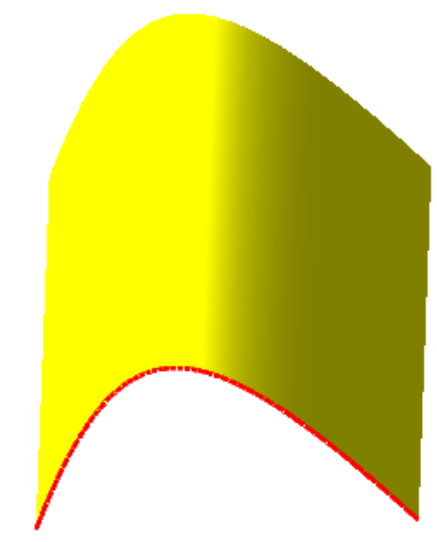

(a) The surface $\boldsymbol{P}(s, t)$ for $\theta(s)=0$

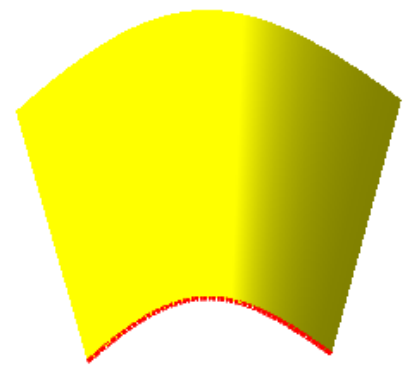

(b) The surface $\boldsymbol{P}(s, t)$ for $\theta(s)=1$

Fig. 5 The spacelike developable surface with spacelike curve.

\section{References}

Alourdas, P. G., Hottel, G. R. and Tuohy, S. T., A design and interrogation system for modeling with rational splines, In: S.K. Chakrabarti et al., eds., Proc. Ninth International Symposium on OMAE, Vol. 1, Part B, Houston, TX, ASME, New York (1990), pp. 555-565.

Aumann, G., Interpolation with developable Bézier patches, Computer Aided Geometric Design, Vol. 8 (1991), pp. 409420.

Bodduluri, R. M. C. and Ravani, B., Design of developable surfaces using duality between plane and point geometries, Computer-Aided Design, Vol. 25 (1993), pp. 621-632.

Chalfant, J. S. and Maekawa, T., Design for manufacturing using B-spline developable surfaces, Journal of Ship Production, Vol. 42 (1998), pp. 207-215.

Chu, C. H. and Séquin, C. H., Developable Bézier patches: properties and design, Computer-Aided Design, Vol. 34 (2002), pp. 511-527.

Do Carmo, M. P., Differential Geometry of Curves and Surfaces, Englewood Cliffs: Prentice Hall (1976).

Kim, Y. H., Yoon, D. W., Ruled surfaces with pointwise 1-type Gauss map, Journal of Geometry and Physics, Vol. 34 (2004), pp. 191-205.

Li, C. Y., Wang, R. H. and Zhu, C. G., Parametric representation of a surface pencil with a common line of curvature, Computer-Aided Design, Vol. 43 (2011), pp. 1110-1117.

Li, C. Y., Wang, R. H. and Zhu, C. G., An approach for designing a developable surface through a given line of curvature, Computer-Aided Design, Vol. 45 (2013), pp. 621-627.

López, R., Differential Geometry of Curves and Surfaces in Lorentz-Minkowski Space, arxiv.org/abs/0810.3351v1 (2008). Martin, R. R., Principal patches-a new class of surface patches based on differential geometry, In: P.J.W. Ten Hagen, ed., Eurographics'83, Proc. 4th Ann. European Association for Computer Graphics Conference and Exhibition, Zagreb, Yugoslavia, North-Holland, Amsterdam (1983), pp. 47-55.

Norlan, T. J., Computer aided design of developable surfaces, Marine Technology, Vol. 8 (1971), pp. $233-242$.

Van de Woestyne, I., Mininal surfaces of the 3-dimensional Minkowski space, Geometry and Topology of Submanifolds II, Singapore; World Scientific, (1990), pp. 344-369.

Walfare, J., Curves and surfaces in Minkowski space (1995), Ph.D. Thesis, K.U. Leuven, Faculty of Science, Leuven. Willmore, T. J., An Introduction to Differential Geometry, Oxford University Press (1959). 